\title{
Contactless vector network analysis using diversity calibration with capacitive and inductive coupled probes
}

\author{
T. Zelder, I. Rolfes, and H. Eul
}

Institut für Hochfrequenztechnik und Funksysteme, Universität Hannover, Appelstraße 9A, 30167 Hannover, Germany

\begin{abstract}
Contactless vector network analysis based on a diversity calibration is investigated for the measurement of embedded devices in planar circuits. Conventional contactless measurement systems based on two probes for each measurement port have the disadvantage that the signal-to-noise system dynamics strongly depends on the distance between the contactless probes.

In order to avoid a decrease in system dynamics a diversity based measurement system is presented. The measurement setup uses one inductive and two capacitive probes. As an inductive probe a half magnetic loop in combination with a broadband balun is introduced. In order to eliminate systematic errors from the measurement results a diversity calibration algorithm is presented. Simulation and measurement results for a one-port configuration are shown.
\end{abstract}

\section{Introduction}

The characterization of single subcircuits within complex planar microwave circuits can be realized by contactless measurement techniques. The measurement of the scattering parameters of each individual subcircuit is useful for the development as well as the quality control of complex microwave planar circuits. Different contactless measurement methods have already been investigated. For example, by Bridges (2004) the scanning probe microscopy, by Osofsky and Schwarz (1992), Gao and Wolff (1997) and Quardirhi and Laurin (2003) contactless electromagnetic probes and by Dudley et al. (1999) electro-optic probes are used to measure the forward and backward traveling waves in front of and behind of the device under test (DUT). Further on, by Hui and Weikle (2005) a non-contacting sampled-line reflectometer is implemented to determine the forward and backward trav-

Correspondence to: T. Zelder

(zelder@hft.uni-hannover.de) eling waves. An overview of different contactless measurement techniques is given by Sayil et al. (2005).

Another contactless measurement approach is based on the contactless vector network analysis. Thereby, the internal directional couplers of a conventional vector network analyzer (VNA) are replaced by contactless probes. For an accurate determination of the scattering parameters conventional calibration algorithms are used. De Groote et al. (2006) and Yhland and Stenarson (2006) applied electromagnetic loops as contactless coupling structures. In contrast to this approach, by Zelder et al. (2007) and Stenarson et al. (2001), pure inductive or capacitive probes are used. A principle setup of a contactless vector network analyzer system using two probes is shown in Fig. 1. The complex planar circuit consists, in this example, of three subcircuits and is fed by the signal source of a two-port vector network analyzer. By means of contactless probes placed in front of and behind of the DUT, a part of the complex signal power is coupled directly to the four receivers of the VNA. In this configuration the internal directional couplers of the VNA are not used. For the elimination of the systematic errors of the measurement setup conventional calibration algorithms can be used as e.g. Short-Open-Load-Thru (SOLT) Schiek (1999) or selfcalibration methods like Thru-Reflect-Line (TRL) Engen and Hoer (1979). One disadvantage of this system is, that for certain probe distances a calibration is not possible which is shown by Zelder et al. (2007). This limits the measurement bandwidth. For the measurement system with two capacitive probes the critical distances have been calculated by Zelder and $\operatorname{Eul}$ (2006).

In this paper the analytical calculation of the critical probe distances is completed for a system with one inductive and one capacitive probe. Further on, to reach a broadband measurement bandwidth, a diversity calibration setup with more than two contactless probes for each measurement port is applied. Simulation and measurement results for a oneport contactless diversity network analyzer based on one

Published by Copernicus Publications on behalf of the URSI Landesausschuss in der Bundesrepublik Deutschland e.V. 


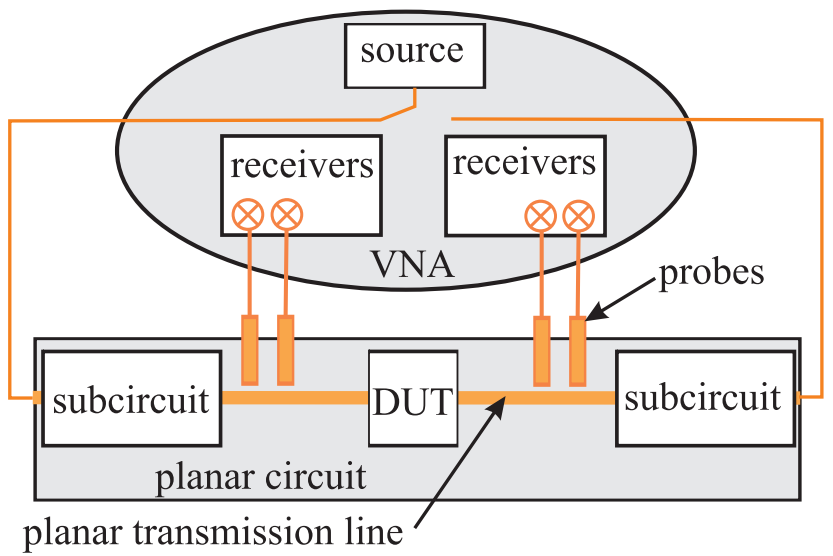

Fig. 1. Principle setup of a contactless vector network analyzer system using two probes for each measurement port.

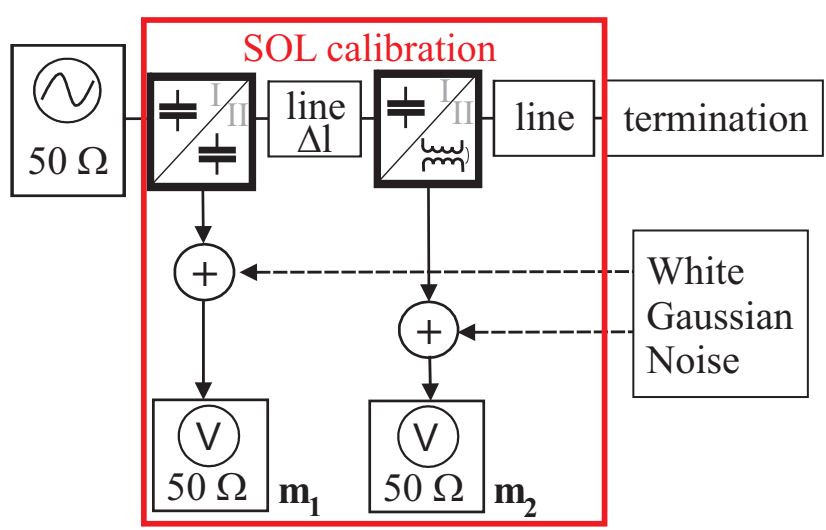

Fig. 2. Simulation model of a contactless measuring system with two electromagnetic probes.

inductive and two capacitive probes are presented. As an inductive probe a half magnetic loop in combination with a balun is used. An extension of the presented method to Nport measurements is possible. In this paper merely the principle function of a contactless diversity based system and its potentials are shown.

\section{Problems using two contactless probes}

The simulation model for a contactless measurement system with two electromagnetic probes is illustrated in Fig. 2. It consists of a signal source with a power level of $1 \mathrm{dBm}$ which feeds a lossless $50 \Omega$ transmission line. As a transmission line termination a calibration standard or the DUT can be used. By means of this model two configurations are considered. In the first configuration, $\mathrm{CC}$, two capacitive probes are used. For the second configuration, LC, one inductive and one capacitive probe are applied. The capacitive coupling is modeled by an ideal capacitance and the inductive

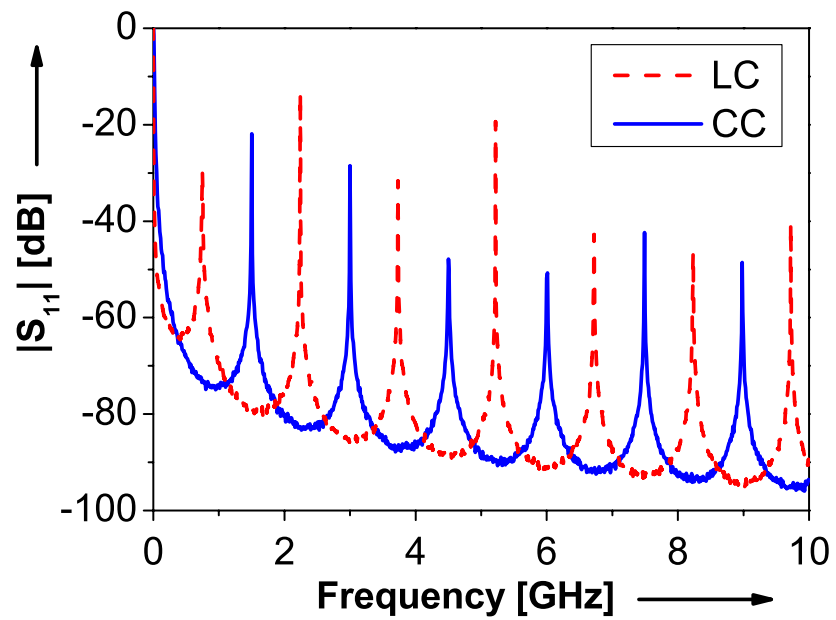

Fig. 3. System dynamics against the frequency for a probe distance of $\Delta \mathrm{l}=100 \mathrm{~mm}$; DUT: $50 \Omega$ load - Data from Zelder and Eul (2006).

coupling by an ideal transformer. The distance between the two probes is $\Delta \mathrm{l}$. The coupled voltages are determined in a $50 \Omega$ system at the receivers $m_{1}$ and $m_{2}$. To get a more realistic system, additive white Gaussian noise is applied at the receivers with a respective power level of $-118 \mathrm{dBm}$ relative to a noise bandwidth of $10 \mathrm{~Hz}$, which is typical for modern vector network analyzers. For the accurate measurement of the scattering parameters of a DUT, the error terms of the system are determined using the conventional Short-OpenLoad (SOL) calibration method Schiek (1999). For this purpose, the complex voltage ratios $\mu_{x}$ of the receivers $m_{1}$ and $m_{2}$ using the three calibration standards short, open and load are measured. In order to verify the calibration and to determine the signal-to-noise system dynamics, the reflection coefficient of a load standard is remeasured. In the following the signal-to-noise system dynamics is abbreviated as system dynamics.

A disadvantage of a contactless system, as illustrated in Fig. 2, is that the system dynamics depends on the distance between the two contactless probes. The system dynamics for two different probe pair combinations is presented in Fig. 3. To show the effect of probe distance, $\Delta 1$ is chosen to $100 \mathrm{~mm}$ which is equivalent to a frequency distance of $3 \mathrm{GHz}$. In Fig. 3 it is shown that at some critical frequencies, which are located in a distance of about $1.5 \mathrm{GHz}$, the dynamics decrease. The reason for these system failures is, that the three voltage ratios $\mu_{x}$ of the two probes measuring the calibration standards short, open and load become equal at these critical frequencies. By contrast, for an accurate calibration the three voltage ratios must be different. Depending on the probe distance, it is possible to determine the critical frequencies analytically, which is shown in detail, for the configuration with one inductive and one capacitive probe (LC), in the next section. 


\section{Analytical analysis of the dynamics decrease using two contactless probes}

At first, the critical probe distances for a contactless one-port measurement system with one inductive and one capacitive probe will be calculated. For the mathematical analysis of the decrease in dynamics range the SOL correction algorithm Schiek (1999) described by Eq. (1) is used.

$$
\frac{\left(\Gamma_{D}-\Gamma_{L}\right) \cdot\left(\Gamma_{S}-\Gamma_{O}\right)}{\left(\Gamma_{D}-\Gamma_{O}\right) \cdot\left(\Gamma_{S}-\Gamma_{L}\right)}=\frac{\left(\mu_{D}-\mu_{L}\right) \cdot\left(\mu_{S}-\mu_{O}\right)}{\left(\mu_{D}-\mu_{O}\right) \cdot\left(\mu_{S}-\mu_{L}\right)}
$$

The reflection coefficients are represented by $\Gamma_{x}$ for the calibration standards short $\left(\Gamma_{S}\right)$, open $\left(\Gamma_{O}\right)$, load $\left(\Gamma_{L}\right)$ and the DUT $\left(\Gamma_{D}\right) . \mu_{x}$ denotes the corresponding ratio of the voltages at the receivers $m_{1}$ and $m_{2}$ :

$\mu_{x}=\frac{U_{m 1}\left(z_{1}\right)}{U_{m 2}\left(z_{2}\right)}$

where $z_{1}$ and $z_{2}$ represent the coupling positions along the transmission line. For the mathematical description of the system dynamics the calibration standards are assumed to be ideal. Thus, the reflection coefficients of the calibration standards are given by:

$\Gamma_{L}=0 ; \Gamma_{S}=-1 ; \Gamma_{O}=1$.

After inserting Eqs. (3) into Eq. (1) the reflection coefficient $\Gamma_{D}$ of the DUT can be calculated:

$\Gamma_{D}=\frac{1}{1-2 \frac{\left(\mu_{D}-\mu_{O}\right) \cdot\left(\mu_{S}-\mu_{L}\right)}{\left(\mu_{D}-\mu_{L}\right) \cdot\left(\mu_{S}-\mu_{O}\right)}}$.

For the mathematical description of the voltage ratios $\mu_{x}$, the voltage $U_{m, V}\left(z_{1}\right)$ obtained by the capacitive probe and the voltage $U_{m, I}\left(z_{2}\right)$ obtained by the inductive probe are considered first. The expressions of the voltages are given by:

$$
\begin{aligned}
U_{m, V}\left(z_{1}\right)= & K_{V} \cdot U_{h}(l)\left(e^{j \varphi\left(z_{1}\right)}\right. \\
& \left.+\Gamma_{n}(l) \cdot e^{-j \varphi\left(z_{1}\right)}\right)+u_{r 1} \\
U_{m, I}\left(z_{2}\right)= & K_{I} \cdot U_{h}(l)\left(e^{j \varphi\left(z_{2}\right)}\right. \\
& \left.-\Gamma_{n}(l) \cdot e^{-j \varphi\left(z_{2}\right)}\right)+u_{r 2}
\end{aligned}
$$

$U_{h}(l)$ represents the voltage amplitude of the forward traveling wave at the position $z=l$ of the planar transmission line. The position $z=l$ indicates the reference plane of the system, $\Gamma_{n}(l)$ represents the reflection coefficient at the reference plane, $K_{V}$ is the voltage coupling coefficient of the capacitive probe and $K_{I}$ is the coupling coefficient of the inductive probe. The terms $u_{r 1}$ and $u_{r 2}$ represent complex noise values. The phase angles of the forward and backward traveling waves are $\pm \varphi$ :

$\varphi=\frac{2 \cdot \pi \cdot f}{v} \cdot(l-z)$

where $f$ represents the frequency and $v$ the phase velocity.
The voltage ratios can be expressed by inserting Eqs. (5) and (6) into Eq. (2):

$\mu_{x}=\frac{K_{V} \cdot U_{h}(l)\left(e^{j \varphi\left(z_{1}\right)}+\Gamma_{x}(l) \cdot e^{-j \varphi\left(z_{1}\right)}\right)+u_{r 1}}{K_{I} \cdot U_{h}(l)\left(e^{j \varphi\left(z_{2}\right)}-\Gamma_{x}(l) \cdot e^{-j \varphi\left(z_{2}\right)}\right)+u_{r 2}}$.

To set up an equation for the voltage ratio $\mu_{L}$, the load reflection coefficient $\Gamma_{L}$ of Eq. (3) is inserted into Eq. (8). With $\varphi\left(z_{1}\right)=\varphi_{1}$ and $\varphi\left(z_{2}\right)=\varphi_{2}$ it results in:

$\mu_{L, L C}=\frac{K_{V} \cdot U_{h}(l) \cdot e^{j \varphi_{1}}+u_{r 1}}{K_{I} \cdot U_{h}(l) \cdot e^{j \varphi_{2}}+u_{r 2}}$.

In order to reduce the arithmetical complexity, the noise contributions for the voltage ratios $\mu_{S}$ and $\mu_{O}$ of the short and open standard are neglected. This results in:

$\mu_{S}=\frac{K_{V} \cdot U_{h}(l)\left(e^{j \varphi_{1}}-1 \cdot e^{-j \varphi_{1}}\right)}{K_{I} \cdot U_{h}(l)\left(e^{j \varphi_{2}}+1 \cdot e^{-j \varphi_{2}}\right)}=j K_{x} \frac{\sin \left(\varphi_{1}\right)}{\cos \left(\varphi_{2}\right)}$

and

$\mu_{O}=\frac{K_{V} \cdot U_{h}(l)\left(e^{j \varphi_{1}}+1 \cdot e^{-j \varphi_{1}}\right)}{K_{I} \cdot U_{h}(l)\left(e^{j \varphi_{2}}-1 \cdot e^{-j \varphi_{2}}\right)}=-j K_{x} \frac{\cos \left(\varphi_{1}\right)}{\sin \left(\varphi_{2}\right)}$

with

$K_{x}=\frac{K_{V}}{K_{I}}$.

For the determination of the system dynamics the load standard is used as a DUT. By use of Eq. (9) we have

$\mu_{D, L C}=\frac{K_{V} \cdot U_{h}(l) \cdot e^{j \varphi_{1}}+u_{r 3}}{K_{I} \cdot U_{h}(l) \cdot e^{j \varphi_{2}}+u_{r 4}}$

with changed noise values $u_{r 3}$ and $u_{r 4}$. Now, the raw data from Eqs. (9) to (13) are inserted into Eq. (4) and it can be transformed to:

$$
\begin{aligned}
\Gamma_{D, L C}= & j \cdot K_{x} \cdot\left(\mu_{D, L C}-\mu_{L, L C}\right) \cos (\Delta \varphi) \\
& \cdot\left[\mu_{L, L C} \cdot \mu_{D, L C} \cdot \sin \left(2 \varphi_{2}\right)\right. \\
& +K_{x}^{2} \cdot \sin \left(2 \Delta \varphi+2 \varphi_{2}\right) \\
& \left.+j \cdot K_{x} \cdot\left(\mu_{L, L C}+\mu_{D, L C}\right) \cos \left(\Delta \varphi+2 \varphi_{2}\right)\right]^{-1} \\
= & N_{L C}(\Delta \varphi) \cdot D_{L C}(\Delta \varphi)^{-1} .
\end{aligned}
$$

The phase difference $\Delta \varphi$ between the two capacitive probes is given by:

$\Delta \varphi=\varphi_{1}-\varphi_{2}$.

A similar analytical calculation can be done using two identical capacitive probes (CC) Zelder and Eul (2006). The dynamics for a contactless measurement system with two capacitive probes results in:

$$
\begin{aligned}
\Gamma_{D, C C}= & \left(\mu_{D, C C}-\mu_{L, C C}\right) \sin (\Delta \varphi) \\
& \cdot\left[\mu_{L, C C} \cdot \mu_{D, C C} \cdot \sin \left(2 \varphi_{2}\right)\right. \\
& +\sin \left(2 \Delta \varphi+2 \varphi_{2}\right) \\
& \left.-\left(\mu_{L, C C}+\mu_{D, C C}\right) \sin \left(\Delta \varphi+2 \varphi_{2}\right)\right]^{-1} \\
= & N_{C C}(\Delta \varphi) \cdot D_{C C}(\Delta \varphi)^{-1} .
\end{aligned}
$$




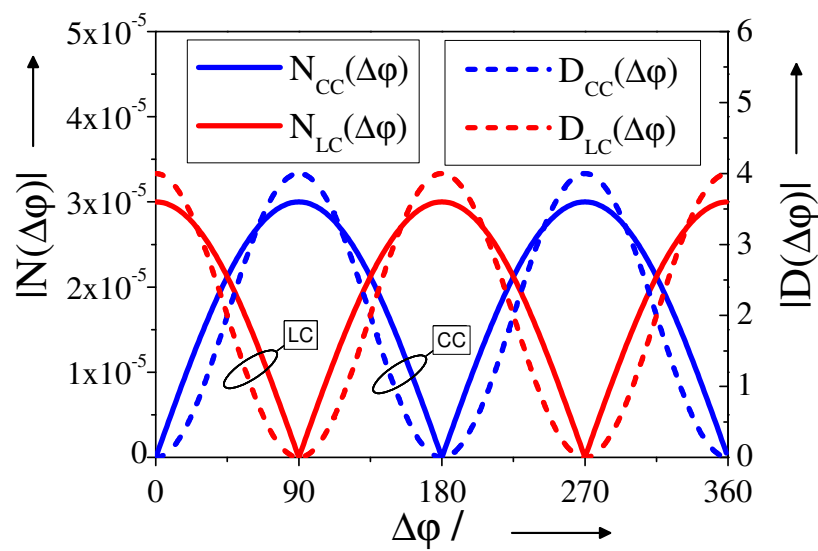

Fig. 4. Calculated results of a contactless measuring system using two probes.

For the determination of the critical probe distances the zeros of the numerator and denominator of Eqs. (14) and (16) must be quantified. Therefore the following simplifications are introduced for the system with two identical capacitive probes:

$$
\begin{aligned}
& \left(\mu_{D, C C}-\mu_{L, C C}\right) \approx \Delta u_{r} \\
& \left(\mu_{D, C C}+\mu_{L, C C}\right) \approx 2 \cdot e^{j \cdot \Delta \varphi} \\
& \left(\mu_{D, C C} \cdot \mu_{L, C C}\right) \approx e^{j \cdot 2 \cdot \Delta \varphi} .
\end{aligned}
$$

Further on, for the measurement system using one inductive and one capacitive probe (LC) the simplifications are as follows:

$$
\begin{aligned}
& \left(\mu_{D, L C}-\mu_{L, L C}\right) \approx K_{x} \cdot \Delta u_{r} \\
& \left(\mu_{D, L C}+\mu_{L, L C}\right) \approx 2 \cdot K_{x} \cdot e^{j \cdot \Delta \varphi} \\
& \left(\mu_{D, L C} \cdot \mu_{L, L C}\right) \approx K_{x}^{2} \cdot e^{j \cdot 2 \cdot \Delta \varphi} .
\end{aligned}
$$

Inserting Eqs. (17) to (19) into Eq. (16) and the Eqs. (20) to (22) into Eq. (14), the dynamics of the contactless measurement system with two contactless probes can be calculated:

$$
\begin{aligned}
& \Gamma_{D, C C}=\frac{2 \cdot j \cdot \Delta u_{r} \cdot \sin (\Delta \varphi) \cdot e^{j \cdot 2 \cdot\left(\Delta \varphi+\varphi_{2}\right)}}{-1+2 \cdot e^{j \cdot 2 \cdot \Delta \varphi}-e^{j \cdot 4 \cdot \Delta \varphi}}=\frac{N_{C C}}{D_{C C}} \\
& \Gamma_{D, L C}=\frac{2 \cdot \Delta u_{r} \cdot \cos (\Delta \varphi) \cdot e^{j \cdot 2 \cdot\left(\Delta \varphi+\varphi_{2}\right)}}{1+2 \cdot e^{j \cdot 2 \cdot \Delta \varphi}+e^{j \cdot 4 \cdot \Delta \varphi}}=\frac{N_{L C}}{D_{L C}} .
\end{aligned}
$$

In Fig. 4 the numerators $N_{C C}, N_{L C}$ and the denominators $D_{C C}, D_{L C}$ of Eqs. (23) and (24) are shown as a function of $\Delta \varphi$. The value of the noise difference $\Delta u_{r}$ is obtained from measurements with a VNA model PNA E8361A. Thereby, the raw data of the reflection coefficient of the $50 \Omega$-load is measured twice in a frequency range between $10 \mathrm{MHz}$ and $20 \mathrm{GHz}$, respectively. The mean value of the difference between the twice measured reflection coefficients is approximately $1.5 \cdot 10^{-5}$, which is used for the analytical investigation. Figure 4 shows distances where the zeros of the numerator and denominator occur are identical for both probe

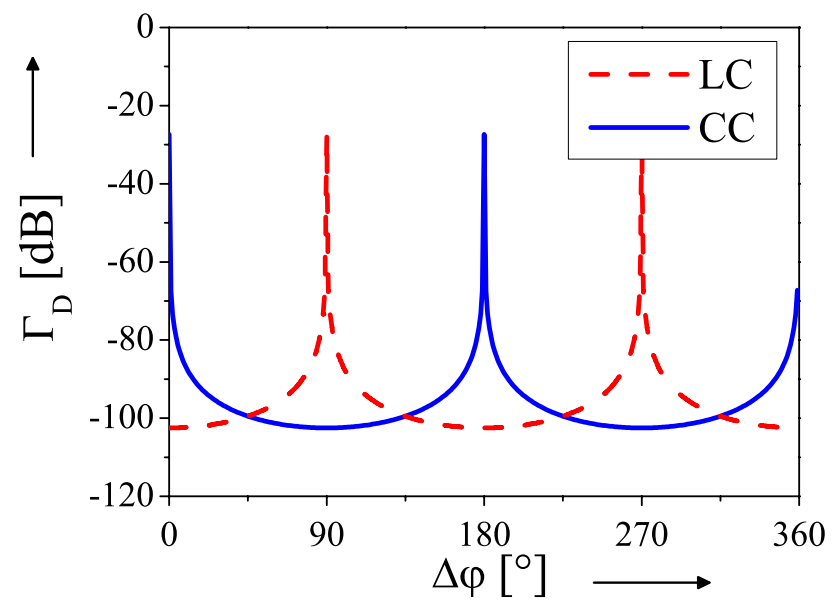

Fig. 5. Calculated reflection coefficients $\Gamma_{D, C C}$ and $\Gamma_{D, L C}$ for both probe pair combinations; DUT: $50 \Omega$ load.

pair configurations. At these probe distances a measurement is not possible. The critical phase differences can be determined by calculating the zeros of the numerators $N_{C C}, N_{L C}$ and the denominators $D_{C C}, D_{L C}$ of Eqs. (23) and (24):

$$
\begin{aligned}
\Delta \varphi_{C C, 0}=m \cdot \pi & \text { with } m=0,1,2,3, \ldots \\
\Delta \varphi_{L C, 0}=n \cdot \frac{\pi}{2} & \text { with } n=1,3,5,7, \ldots
\end{aligned}
$$

For these phase differences the system dynamics decreases. The characteristics of the system dynamics is illustrated in Fig. 5. At the critical phase differences $\Delta \varphi$, peaks in the system dynamics occur, so that a measurement is not possible. For two capacitive probes a calibration is not possible, if the distance is in the range of integer multiples of $\lambda / 2$. In case of one inductive and one capacitive probe the critical distances between the probes are odd-numbered multiples of $\lambda / 4$. For a given probe distance $\Delta l$ and for an effective permittivity $\epsilon_{r \text {,eff }}$ of the planar transmission line we have the critical probe frequencies:

$$
\begin{aligned}
& f_{\text {crit }, C C}=m \cdot \frac{c_{0}}{2 \cdot \sqrt{\epsilon_{r, \text { eff }}} \cdot \Delta l} \\
& f_{\text {crit }, L C}=n \cdot \frac{c_{0}}{4 \cdot \sqrt{\epsilon_{r, \text { eff }}} \cdot \Delta l}
\end{aligned}
$$

\section{Simulation model of a contactless diversity measure- ment system using different probe types}

For contactless measurements using two probes for each port of the DUT, the measurement bandwidth is limited due to the probe distance, as shown in Sects. 2 and 3. By means of a diversity based measurement system a large measurement bandwidth can be achieved. For a contactless diversity measurement setup, more than two contactless probes for each port of the DUT are used. One-port measurement results using such a diversity based system are given by Zelder and Eul 


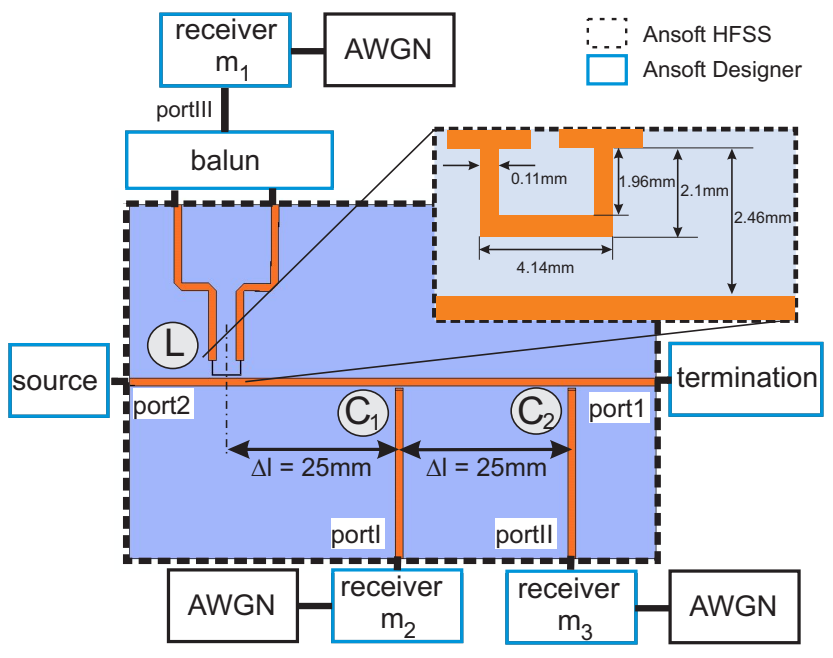

Fig. 6. Simulation model for the basic analysis of the contactless diversity system.

(2006). There, three identical contactless capacitive probes made from very small semi-rigid coaxial lines with an outer diameter of $86 \mu \mathrm{m}$, are applied.

In this paper, a diversity based measurement setup using two identical capacitive $\left(C_{1}, C_{2}\right)$ and one inductive probe $(L)$ is examined. At first, a simulation of the system is performed. The simulation model is given in Fig. 6. A continuous wave of a power level of $1 \mathrm{dBm}$ is led by a signal source to a microstrip line at port 2. During the calibration and measurement the signal is swept over the frequency. The width of the first microstrip line is $1.12 \mathrm{~mm}$ equivalent to a line impedance of $50 \Omega$. As terminations of the planar transmission line, the calibration standards short, open, load as well as a DUT are used. Without loss of generality, the three contactless probes are realized on the same substrate (Rogers 4003) as the microstrip line. Of course, it is also possible to use separated probes. To illustrate the principal diversity method and its potentials, this setup is sufficient. For the setup all distances between the probes are chosen to $\Delta \mathrm{l}=25 \mathrm{~mm}$. The size of the substrate is $76.2 \mathrm{~mm} \times 50.8 \mathrm{~mm}$.

In the setup the two capacitive probes are modeled by means of a second and third microstrip line, which are not connected to the main line. The distance between the capacitive probes and the main microstrip line is $300 \mu \mathrm{m}$. The inductive probe is realized by a half magnetic loop. The geometry of the loop is also given in Fig. 6. The loop is directly connected to two additional microstrip lines. One characteristic of a half loop is that the energy is coupled through the loop by the electric and magnetic field at the main microstrip line. To get rid of the capacitive coupled voltage, a balun is used at the end of the loop. The ports of the two capacitive probes and the inductive probe are denoted by port I, port II and port III. The return losses of the main microstrip line are given in Fig. 7 and the coupling factors between the first mi-

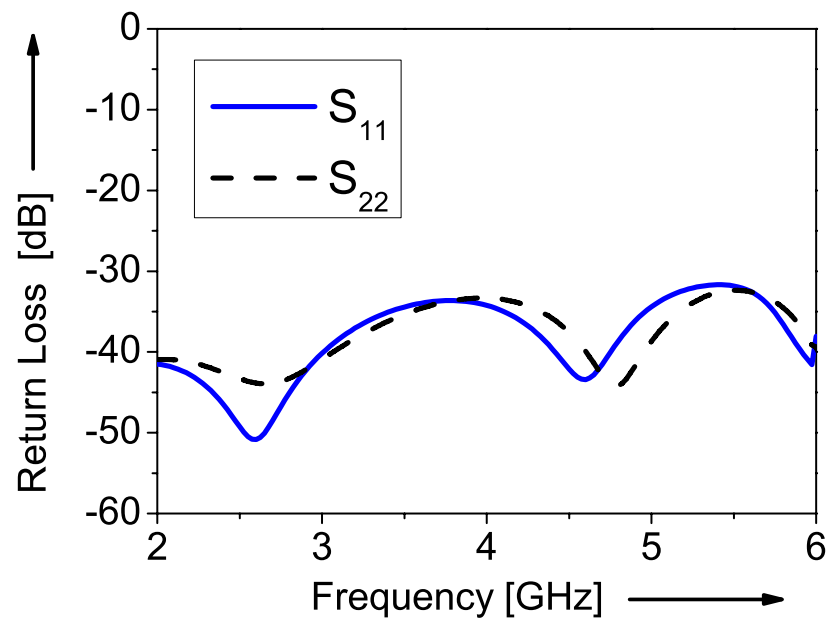

Fig. 7. Return Loss of the main microstrip line for port 1 and port 2.

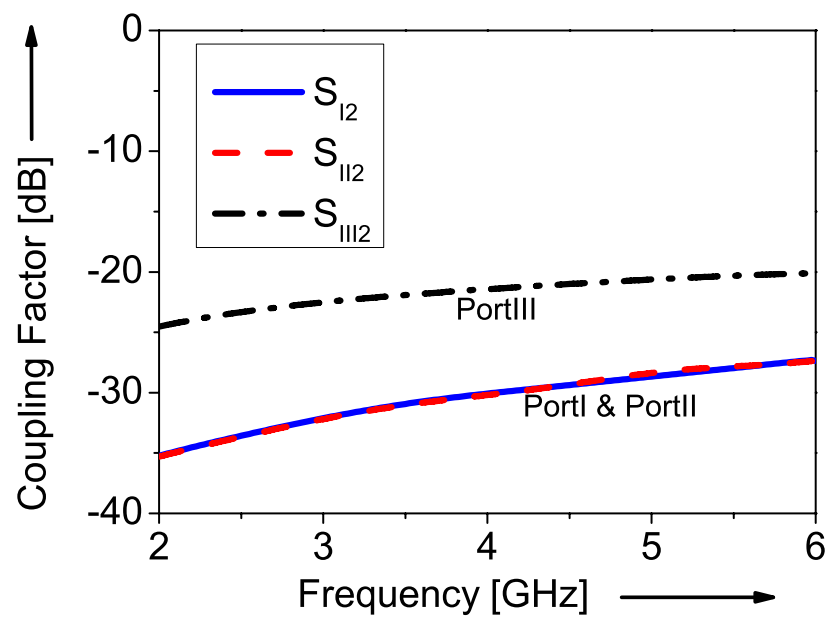

Fig. 8. Coupling factor between port 2 of the first microstrip line and port I, port II and port III.

crostrip line and the probes are illustrated in Fig. 8. For the simulation of this setup two different programs were considered. To determine the scattering parameters of the planar six-port circuit, HFSS ${ }^{\mathrm{TM}}$ of Ansoft was used. The other elements like the balun, the receivers, the source and the terminations were simulated using ANSOFT DESIGNER. Introducing white Gaussian noise, the system was calibrated by using the diversity SOL (DSOL) calibration algorithm which was implemented in MATLAB. A description of the DSOL calibration is given in the next section.

\section{Diversity SOL calibration}

For a better understanding of the diversity calibration, a general block diagram of the probe pair selection is given in 


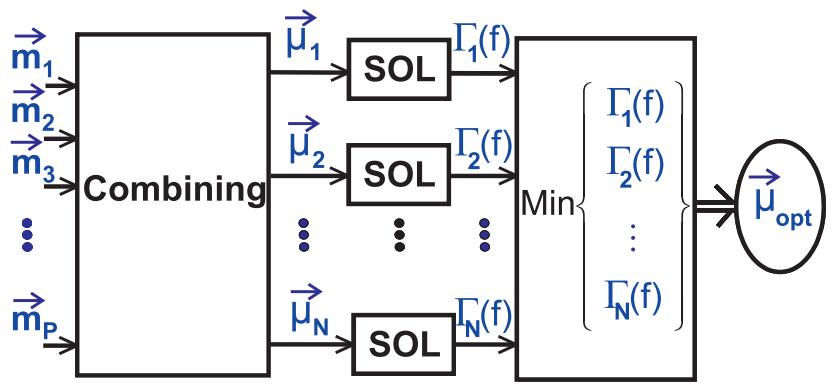

Fig. 9. General block diagram of the probe pair selection.

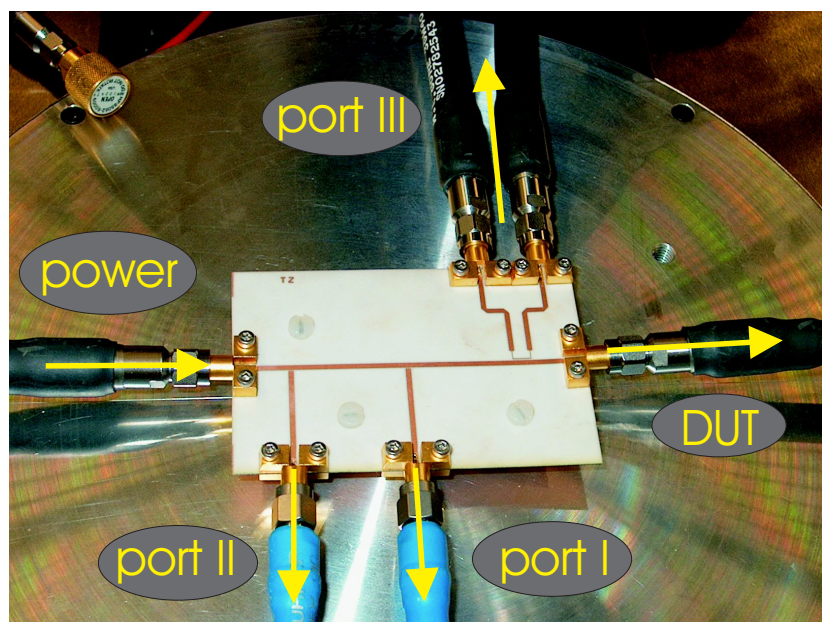

Fig. 10. Photograph of the contactless measurement setup with three probes.

Fig. 9. The input quantities of the algorithm are the frequency dependent, complex voltage vectors $\boldsymbol{m}_{1}$ to $\boldsymbol{m}_{P}$ of the $P$ contactless probes. Each voltage vector $\boldsymbol{m}_{p}$ consists of four voltage values resulting from the measurement of the calibration standards short, open and load with $U_{m_{p}, S}$, $U_{m_{p}, O}, U_{m_{p}, L}$ and the DUT with $U_{m_{p}, D}$ for the probe $p$ :

$\boldsymbol{m}_{p}=\left[U_{m_{p}, S}(f) ; U_{m_{p}, O}(f) ; U_{m_{p}, L}(f) ; U_{m_{p}, D}(f)\right]$.

In order to determine the system dynamics, the load standard is employed also as the DUT.

The voltage ratios $\boldsymbol{\mu}_{n}$ for each possible probe pair combination are calculated using all measured probe voltages. With $P$ contactless probes, $N$ different probe pair combinations are possible. $N$ is given by the binomial coefficient:

$N=\left(\begin{array}{l}P \\ 2\end{array}\right)=\frac{P !}{2 \cdot(P-2) !}$.

One example of the voltage ratio $\mu_{n}$ for the nth probe pair combination using the probes $p_{1}$ and $p_{2}$ is given by:

$$
\begin{aligned}
\boldsymbol{\mu}_{n} & =\left[\frac{U_{m_{p_{1}}, S}}{U_{m_{p_{2}}, S}} ; \frac{U_{m_{p_{1}}, O}}{U_{m_{p_{2}}, O}} ; \frac{U_{m_{p_{1}}, L}}{U_{m_{p_{2}}, L}} ; \frac{U_{m_{p_{1}}, D}}{U_{m_{p_{2}}, D}}\right] \\
& =\left[\mu_{n, S} ; \mu_{n, O} ; \mu_{n, L} ; \mu_{n, D}\right]
\end{aligned}
$$

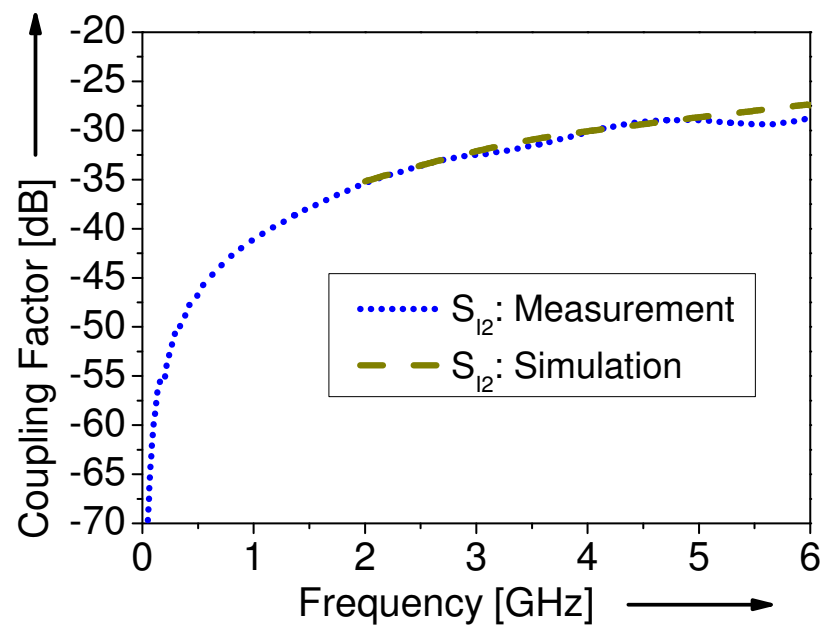

Fig. 11. Comparison of the coupling factors of the simulation and the measurement results.

With the knowledge of all voltage ratios $\mu_{1}$ to $\mu_{N}$ the reflection coefficients $\Gamma_{1}$ to $\Gamma_{N}$ for all possible probe pair combinations are determined using the conventional SOL correction algorithm of Eq. (4). As the load standard is used as DUT, the determined reflection coefficients $\Gamma_{1}$ to $\Gamma_{N}$ describe the system dynamics. In order to get, for each frequency, the probe pair combinations showing the highest dynamics the minima of the reflection coefficients have to be found. With these minima, the best probe pair combinations are known for which the largest system dynamics can be achieved. These probe pairs are used for further measurements of an unknown DUT. The described diversity combining can also be implemented with other calibration methods like SOLT, TRL, etc. For a practical test, a real diversity measurement setup is discussed in the next section.

\section{Measurement setup}

The one-port measurement system is realized according to the simulation setup of Fig. 6. The setup is shown in Fig. 10. The line is fed by the signal source of the VNA of a power level of $-14 \mathrm{dBm}$. The forward and backward traveling signals propagating on the main line are partly coupled to three coherent receivers of the VNA, by two capacitive probes and one inductive probe. The geometries of the inductive and capacitive coupling structures are described in Sect. 4. The measured coupling factors of the capacitive probes are approximately $-35 \mathrm{~dB}$ at $2 \mathrm{GHz}$ and $-25 \mathrm{~dB}$ at $10 \mathrm{GHz}$ and fit very well to the simulated values. As an example, the simulated and measured coupling factors between port I and port 2 are compared in Fig. 11. The difference of measurement and simulation can be explained by influence of microstrip to coaxial transitions used in the measurement. These were connected to coaxial cables used to feed the 
Table 1. Critical frequencies.

\begin{tabular}{ccc}
\hline$f_{L C 1}[\mathrm{GHz}]$ & $f_{L C 2}[\mathrm{GHz}]$ & $f_{C 1 C 2}[\mathrm{GHz}]$ \\
\hline 1.85 & 0.92 & 0.00 \\
5.54 & 2.77 & 3.69 \\
9.23 & 4.62 & 7.39 \\
12.92 & 6.46 & 11.08 \\
$\vdots$ & $\vdots$ & $\vdots$ \\
\hline
\end{tabular}

device and to connect the DUT and precise coaxial calibration standards at a well-defined reference plane. The obtained simulation and measurement results are given in Sect. 7.

\section{Results}

\section{$7.150 \Omega$ standard}

A comparison of the system dynamics for the simulation model and the measurement system using the $50 \Omega$ calibration load as DUT is shown in Figs. 12 and 13. The results show a good accordance. The remaining differences are due to fabrication tolerances of the coupling substrate and to the constant noise level used in the simulation. According to a $10 \mathrm{~Hz}$ intermediate frequency bandwidth of the PNA E8361A, reference AWGN was applied in the simulation with a respective power level of $-118 \mathrm{dBm}$. For example, Fig. 12 shows the system dynamics using the probe pair combination $L$ and $C_{2}$. The system was calibrated with a conventional SOL algorithm. Here peaks occur showing at which frequencies the calibration fails and consequently where accurate measurements are not possible. By knowledge of the probe distances and of the mean value of the effective permittivity of the main microstrip line, which is $\epsilon_{r, \text { eff }}=2.64$ at $6 \mathrm{GHz}$, the critical frequencies can be calculated using Eqs. (27) and (28). The results are given in Table 1 . The calculated critical frequencies $f$ for the three possible probe pair combinations are equal to the frequencies obtained by simulation and measurement. In comparison to the system using two contactless probes, the diversity system achieves a broad measurement bandwidth. Critical frequencies do not exist any more. Figure 13 shows the achieved measurement bandwidth of the diversity based system. The system dynamics is approximately $-70 \mathrm{~dB}$. Compared to the $L C_{2}$ combination (Fig. 12), a gain of about $30 \mathrm{~dB}$ is achieved.

\subsection{Open standard}

Here a high reflective DUT is considered, realized by the open calibration standard. In Fig. 14, the measured reflec-

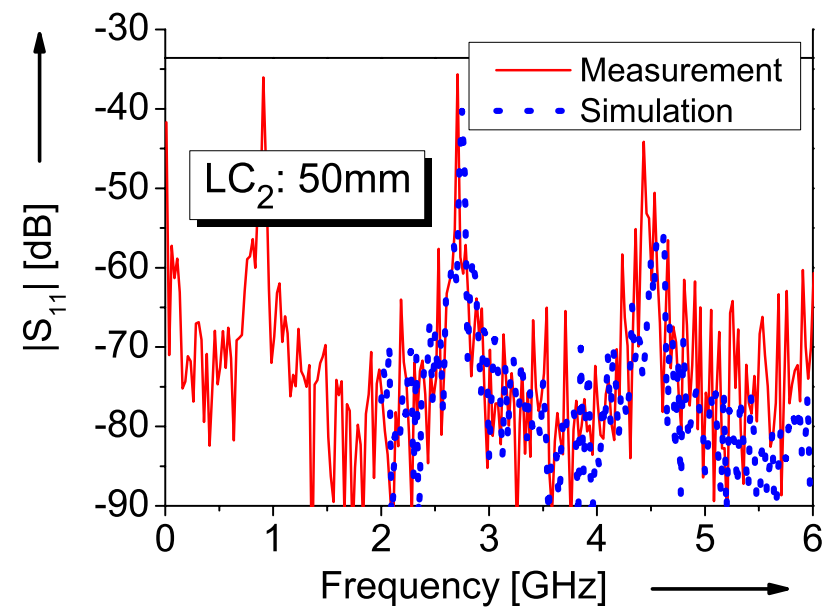

Fig. 12. Comparison of simulated and measured system dynamics for combination $\mathrm{LC}_{2}$.

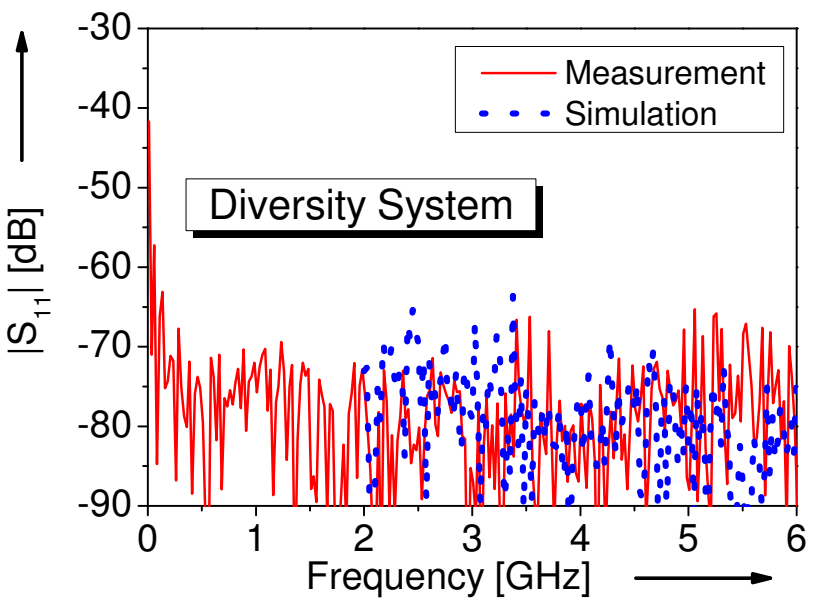

Fig. 13. Comparison between the simulated and measured system dynamics for the diversity system.

tion coefficients are given for all three possible probe pair combinations $L C_{1}, L C_{2}$ and $C_{1} C_{2}$. A SOL calibration was applied. We observe, that at the critical frequencies given in Table 1, peaks occur with an amplitude of up to $0.3 \mathrm{~dB}$. An improvement can be achieved using the diversity measurement system. The measurement setup of Fig. 10 is calibrated using the DSOL calibration, and then the open calibration load is remeasured. The results are shown in Fig. 15. Compared with the results given in Fig. 14, a significant improvement can be achieved: Only small peaks with a maximum amplitude of $0.04 \mathrm{~dB}$ exist.

7.3 Comparison of contactless and conventional vector network analysis using an offset-load

The contactless system using two probes and the diversity based system was compared here using a conventional 


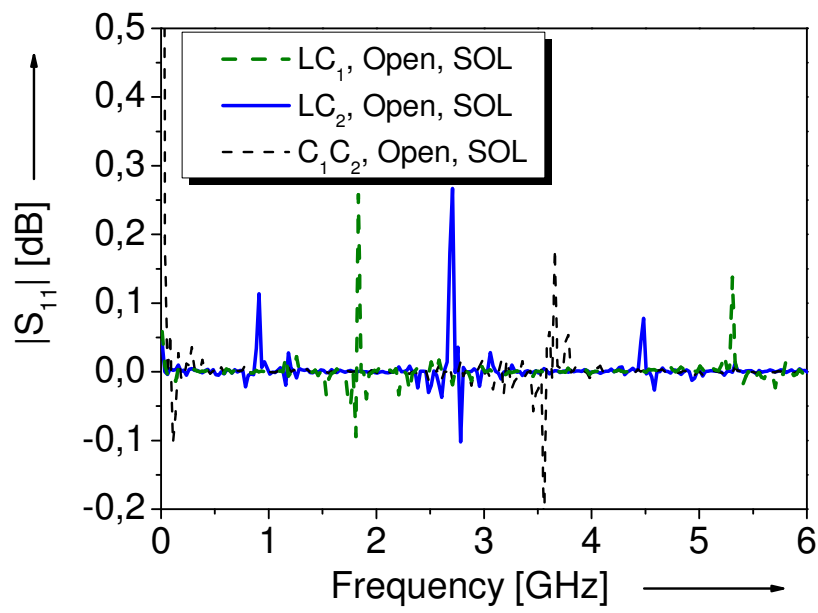

Fig. 14. Measurements results of the open standard as a DUT, after a SOL calibration, for three probe pair combinations.

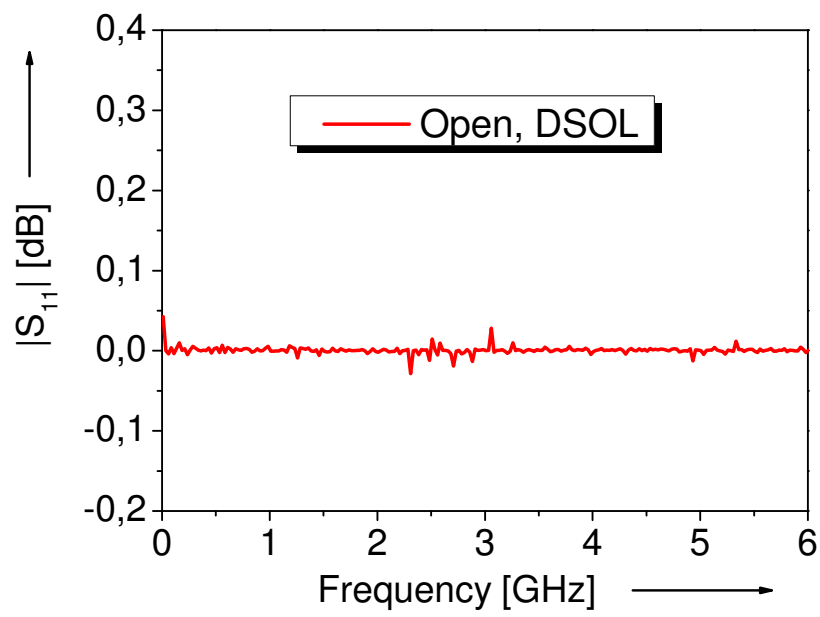

Fig. 15. Measurements results of the open standard as a DUT, after a DSOL calibration, for the diversity system.

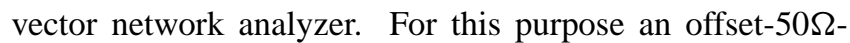
load, which is not used for the calibration, is applied as a DUT. The results are shown in Figs. 16 to 19. Thereby, the measurement data from a conventional vector network analysis was used as a reference.

In particular Fig. 16 shows the percentage deviations of the absolute values of the measured reflection coefficients using the system with the probe pair combinations $L C_{1}, L C_{2}$ and $C_{1} C_{2}$. Thereby, the amplitude errors (AE) of the contactless systems were calculated by using Eqs. (32):

$A E=100 \% \cdot \frac{\left|\Gamma_{\text {contactless }}\right|-\left|\Gamma_{\text {conventional }}\right|}{\left|\Gamma_{\text {conventional }}\right|}$.

At the critical frequencies large errors in the characteristics of the measured reflection coefficients occur. These result in measurement errors of up to $670 \%$ between $35 \mathrm{MHz}$

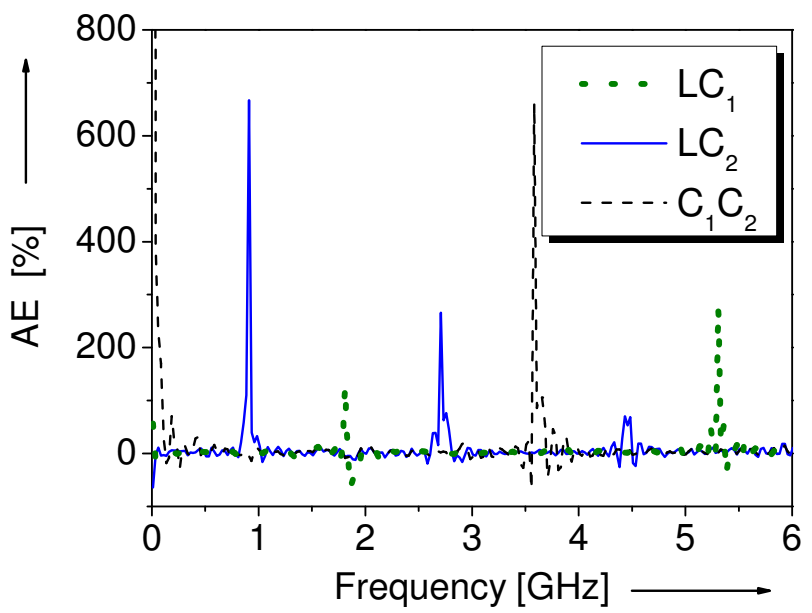

Fig. 16. Deviations between the contactless system with two probes and the measurement results using the conventional VNA, DUT:

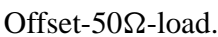

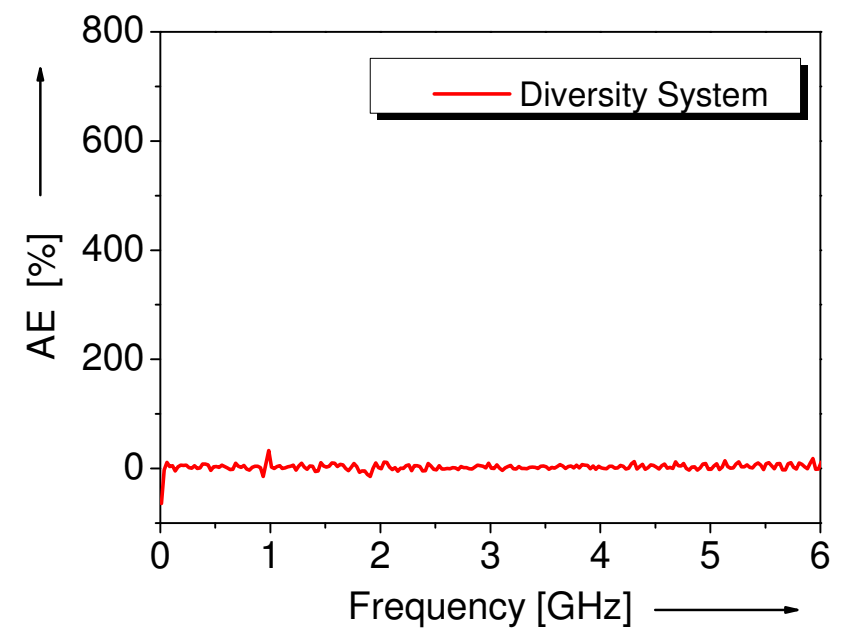

Fig. 17. Deviations between the contactless diversity based system with three probes and the measurement results using the conven-

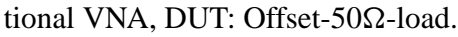

and $6 \mathrm{GHz}$. In contrast to these results, the errors are minimized using the diversity based system. The described diversity based system shows a significant improvement of the contactless measurement system, as can be seen in Fig. 17. The results of the diversity based system show a maximal error of $32 \%$. The remaining maximal error seems to be quite high, but it has to be annotated that for the calculation of the percentage deviation a very small reference value of about $-35 \mathrm{~dB}$ of the offset load is used.

Considering the phase, for the contactless system of two probes, phase errors of up to $178^{\circ}$ occur at the critical frequencies. By means of the diversity based system a maximal error of $22^{\circ}$ arises in the frequency range between $35 \mathrm{MHz}$ and $6 \mathrm{GHz}$, as shown in Figs. 18 and 19. Thus, a clear 


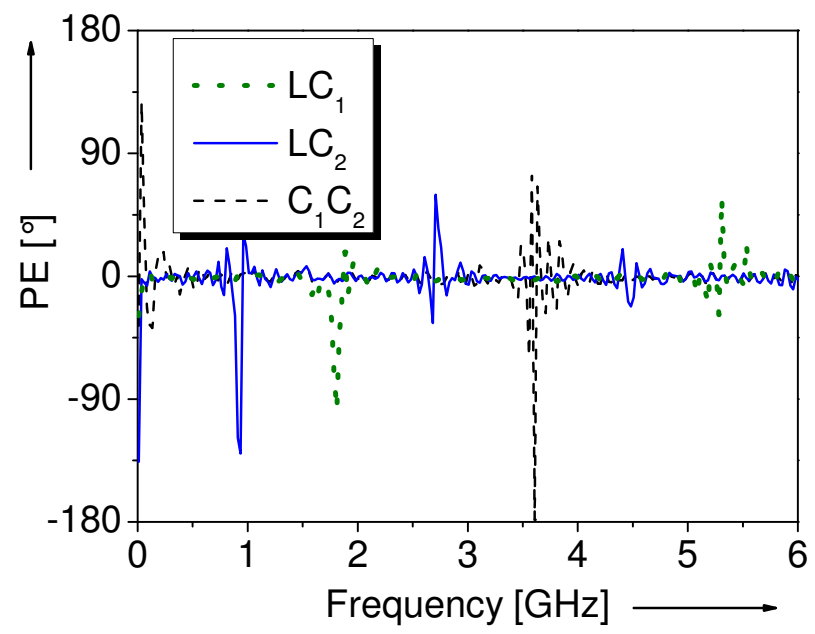

Fig. 18. Deviations between the contactless diversity based system with three probes and the measurement results using the conven-

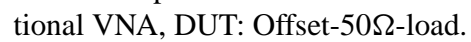

reduction of the measurement errors can be achieved. The phase errors (PE) were calculated by using Eqs. (33):

$P E=\arg \left(\Gamma_{\text {contactless }}\right)-\arg \left(\Gamma_{\text {conventional }}\right)$.

A part of the remaining errors of the diversity system are caused by non-perfect reproducibility of the system and by cross-coupling of signals in the planar structure. An improvement of the measurement accuracy can be achieved by using shielded probes, which is shown by Zelder et al. (2007).

\section{Conclusions}

In the first part of this paper the contactless vector network analysis using two probes is presented. It is shown that dependent on the distance between the two probes, a calibration is not possible at certain frequencies. For the two configurations using either two capacitive probes or one inductive and one capacitive probe, these critical frequencies are obtained analytically. An enhanced, diversity based, contactless measurement system is presented which eliminates these system failures. In a one-port setup two capacitive and one inductive probe are integrated in a system. A diversity calibration algorithm is applied to determine probe pair combinations, for which the largest system dynamics can be achieved. The most convenient probe pair combinations are then used for the measurement of the reflection coefficient of a DUT. Simulation and measurement results show a significant improvement of the system dynamics of up to $30 \mathrm{~dB}$. It is also illustrated that when using two probes, peaks of up to $0.3 \mathrm{~dB}$ in the return loss occur in case of a high-reflective DUT, which are avoided using the contactless diversity based system. Further on, the reflection of an offset-load was determined using a conventional vector network analyzer. The

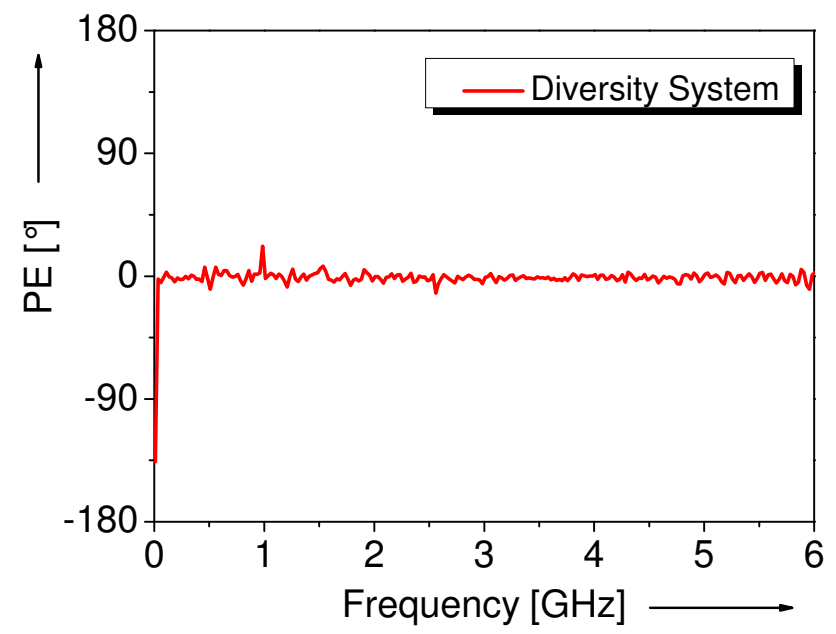

Fig. 19. Deviations between the contactless diversity based system with three probes and the measurement results using the conven-

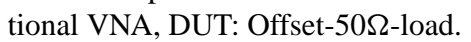

results are compared with reflection measured by the contactless system using two probes and by the contactless diversity based system. And in this case, the measurement errors can be reduced significantly by applying the diversity based system.

\section{References}

Bridges, G. E.: Non-contact probing of integrated circuits and packages, IEEE MTT-S International Microwave Symposium Digest, vol. 3, pp. 1805-1808, June, 2004.

De Groote, F., Verspecht, J., Tsironis, C., Barataud, D., and Teyssier, J.-P.: An improved coupling method for time domain load-pull measurements, European Microwave Conference, vol. 1, October, 2005.

Dudley, R. A., Roddie, A. G., Bannister, D. J., Gifford, A. D., Krems, T., and Facon, P.: Electro-optic S-parameter and electricfield profiling measurement of microwave integrated circuits, IEE Proceedings Science, Measurement and Technology, vol. 146, no. 3, pp. 117-122, May, 1999.

Engen, G. F. and Hoer, C. A.: Thru-Reflect-Line: An improved technique for calibrating the dual six-port automatic network analyzer, IEEE Transaction on Microwave Theory and Techniques, vol. 12, pp. 987-993, December, 1979.

Gao, Y. and Wolff, I.: Measurements of field distributions and scattering parameters in multiconductor structures using an electric field probe, IEEE MTT-S International Microwave Symposium Digest, vol. 3, pp. 1741-1744, June, 1997.

Hui, D. and Weikle, R. M.: A non-contacting sampled-line reflectometer for microwave scattering parameter measurements, 64th ARFTG Microwave Measurements Conference, Fall 2004, pp. 131-137, Dec., 2004.

Osofsky, S. S. and Schwarz, S. E.: Design and performance of a noncontacting probe for measurements on high-frequency planar circuits, IEEE Transactions on Microwave Theory and Techniques, vol. 40, no. 8, pp. 1701-1708, Aug., 1992. 
Quardirhi, Z. and Laurin, J. J.: Méthode de measures des paramétres s sans contact, CCECE 2003-CCGEI 2003, Montréal, May, 2003.

Sayil, S., Kerns, D. V., and Kerns, S. E.: A survey contactless measurement and testing technique potentials, IEEE Potentials, vol. 24, no. 1, pp. 25-28, February-March, 2005.

Schiek, B.: Grundlagen der Systemfehlerkorrektur von Netzwerkanalysatoren, Grundlagen der Hochfrequenz-Messtechnik, 1th ed., Berlin Heidelberg, Germany, Springer-Verlag, chapter 4, pp. 141-174, 1999.

Stenarson, J., Yhland, K., and Wingqvist, C.: An in-circuit noncontacting measurement method for S-parameters and power in planar circuits, IEEE Transactions on Microwave Theory and Techniques, vol. 49, no. 12, pp. 2567-2572, December, 2001.
Yhland, K. and Stenarson, J.: Noncontacting measurement of power in microstrip circuits, in 65th ARFTG, pp. 201-205, June, 2006.

Zelder, T., Rabe, H., and Eul, H.: Contactless electromagnetic measuring system using conventional calibration algorithms to determine scattering parameters, Adv. Radio Sci., 5, 2007.

Zelder, T. and Eul, H.: Contactless network analysis with improved dynamic range using diversity calibration, Proceedings of the 36th European Microwave Conference, Manchester, UK, pp. 478-481, September, 2006. 